\title{
Converse Trading Strategies, Intrinsic Noise and the Stylized Facts of Financial Markets
}

\author{
Frank Westerhoffa,* Reiner Franke ${ }^{\mathrm{b}}$ \\ April 2009 \\ ${ }^{a}$ University of Bamberg, Germany \\ ${ }^{\mathrm{b}}$ University of Kiel, Germany
}

\begin{abstract}
The paper proposes a simple asset pricing model with three groups of traders: chartists who believe in the persistence of bull and bear markets, fundamentalists who bet on a reduction of the observed mispricing, and investors who follow a buy and hold strategy. The innovative feature of the model concerns the frequency of trading: rather than remaining constant over time, each agent in a group is only assumed to become active with a certain probability over a given market period. Depending on the trading strategies, part of this elementary kind of intrinsic noise is additive and another part is multiplicative. Using bootstrap and Monte Carlo methods, it is demonstrated that this combination can contribute to explaining the stylized facts of the daily returns on financial markets, such as volatility clustering, fat tails, and the autocorrelation patterns.
\end{abstract}

JEL classification: D84; G12; G14; G15.

Keywords: Fundamentalist and technical trading; model validation; volatility clustering; daily returns; autocorrelation patterns.

\section{Introduction}

Models with heterogeneous agents that rely on simple heuristic trading strategies have proven to be quite successful in generating interesting dynamics that more or less resem-

\footnotetext{
* Corresponding author.

Email address: frank.westerhoff@uni-bamberg.de (Frank Westerhoff).
} 
ble the evolution of asset prices on financial markets. ${ }^{1}$ Guided by questionnaire evidence (Menkhoff and Taylor, 2007), this literature focusses on the behaviour of fundamental and technical traders. The latter, also called chartists, employ trading methods that attempt to extract buying and selling signals from past price movements (Murphy, 1999). Fundamentalists, by contrast, bet on a reduction of the current mispricing with respect to some fundamental value of the asset (see already Graham and Dodd, 1951). Despite the many different specifications of the fundamental and technical trading rules, the interplay of the counteracting forces to which they give rise is the basic mechanism driving the price dynamics in these models.

An important branch of the research is concerned with time-varying population shares of the heterogeneous agents. In Brock and Hommes (1998), for instance, agents switch between chartism and fundamentalism according to an evolutionary fitness measure reflecting their past trading performance. In Kirman (1991), social interactions between heterogeneous agents can cause swings in opinion, e.g. a major shift from fundamental analysis to technical analysis. In Lux and Marchesi (1999), agents tend to follow the crowd but also compare the recent success of their trading strategies. In a nutshell, these models work as follows. Consider a situation where technical traders dominate the market. The dynamics is then likely to become unstable and stronger price fluctuations, associated with bubbles and crashes, may emerge. Eventually, however, fundamental analysis may increase in popularity for reasons that can differ from one model to another. This tends to stabilize the market and restore tranquillity. Nevertheless, after some time the prospects of technical trading improve again, and the process repeats itself in an intricate manner.

Numerous stochastic versions of such scenarios are more ambitious in that they attempt to mimic certain stylized facts of the financial markets. Concentrating on daily data, a first challenge in calibrating these models is to produce the uncorrelated price changes typically observed in reality. One way to achieve this is to build large-scale models with many different and sometimes even endogenously evolving trading strategies (LeBaron 2006). Given such a large and diverse ecology of competing trading strategies, autocorrelations in returns are liable to be cancelled out. A problematic feature of this generally quite appealing approach is that one may end up with a black box, and pinning down the main causalities in the many dynamic feedbacks is an arduous task. An alternative method is to construct small-scale models with only a few (two or three) trading strategies (Hommes, 2006). One disadvantage of this approach is that it requires the modeller to impose a substantial amount of exogenous noise on some of the equations in order to eliminate the autocorrelations between returns (see Westerhoff and Dieci, 2006, for a particular example), apart from the problem of simultaneously matching some ad-

${ }^{1}$ For recent surveys of this burgeoning field of research, see Chiarella et al. (2009), Hommes and Wagener (2009), Lux (2009a) and Westerhoff (2009), among others. 
ditional features of financial markets. On the other hand, small-scale models are perhaps analytically tractable and their dynamic mechanisms are easy to understand.

Against this background, our contribution to this literature can be briefly sketched as follows. First, the structural model we put forward falls into the category of (very) simple models. Second, random forces are vital to our price dynamics, too. However, instead of adding them exogenously, we can interpret them as intrinsic stochastic noise. Our innovation in this respect is that we do not fix the number of active traders of a given type but let them vary stochastically from one market period to another. We believe that variable trading frequencies are a relevant phenomenon of the real world, and not abstracting from them will prove to be a fruitful extension of existing models.

Third, to make our point as stark, or pure, as possible, the fundamentalists and technical traders are specified in such a way that their individual asset demands directly oppose each other, and that the noise originating from their time-varying market participation is multiplicatively linked to the price. Furthermore, we introduce another type of demand that gives rise to additive noise. As it turns out, the combination of these most elementary mechanisms has the potential to reproduce the main stylized facts of the daily returns on the financial markets - not perfectly but to a remarkable degree. To be more precise, we are interested here in the absence of autocorrelations in the raw returns, in the fat tails of their frequency distributions, and in volatility clustering and long memory effects.

The remainder of the paper is organized as follows. The model is formulated in the next section. Section 3 presents a typical simulation run. It serves to discuss the functioning of the model and also contrasts various properties of its return series with their empirical counterparts from the S\&P 500 stock market index. In Section 4 the robustness of these features is tested. To this end, the frequency distributions of a set of selected summary statistics from a battery of Monte Carlo experiments are set against the frequency distributions that are obtained from the same number of bootstrap samples of the empirical returns. We view these investigations as a validation of the model with respect to the daily observations from major stock market indices. In a similar study it is shown in Section 5 that the model is able to discriminate between stock and foreign exchange markets (specifically, the USD-DEM exchange rate). Section 6 concludes.

\section{Formulation of the model}

We consider a market for one risky asset that is populated by three kinds of traders, whom we call fundamentalists, chartists, and investors. Fundamentalists base their demand on the differences between the current price and the fundamental value. Even though they might expect the gap between the two prices to widen in the immediate future, they do not trade on the likeliness of this event and rather choose to place their bets on an eventual rapprochement. Chartists, on the other hand, believe in the persistence of a bull 
or bear market over the next few days and accordingly buy in the first case and sell in the second. Thus they formally take the opposite view of the fundamentalists. In fact, although it is not necessary to specify fundamentalism and chartism in this model simply as two converse trading strategies, it is nevertheless very convenient for our purposes. ${ }^{2}$

These agents, however, are not active in each and every period, nor is it a constant fraction of each group that enters the market. If a single chartist or fundamentalist, respectively, becomes active on day $t$, then, neglecting any nonlinearities, his or her market orders $d_{t}^{C}$ and $d_{t}^{F}$ are supposed to be given by

$$
\begin{aligned}
& d_{t}^{C}=\chi\left(p_{t}-p^{\star}\right) \\
& d_{t}^{F}=\phi\left(p^{\star}-p_{t}\right)
\end{aligned}
$$

where $p_{t}$ denotes the log price of the asset, $p^{\star}$ its (constant) fundamental value, and $\chi$ and $\phi$ are two constant and positive coefficients that measure the agents' responsiveness to the observed mispricing. ${ }^{3}$

The third group of agents, the investors, do not explicitly act on the price but trade for reasons outside the model. Occasionally such an agent buys the asset to hold it for a longer time, and with equal probability an exogenous event induces him or her to sell the asset in his/her possession; one motivation here, for example, can be concerns about liquidity. Formally, a pool of potential buyers and a pool of potential sellers may be distinguished, where an active agent buys or sells $d_{t}^{B}$ and $d_{t}^{S}$ units of the asset, respectively. In order to avoid a drift in the price (which would also affect the fundamental value), we postulate already at the present stage that this number is identical for both groups. It is also fixed at a positive value $\kappa>0$, leading to

$$
\begin{aligned}
& d_{t}^{B}=\kappa \\
& d_{t}^{S}=-\kappa
\end{aligned}
$$

To make the exposition easier, we admit that a given agent can be in the pool of potential buyers at some time and in the pool of potential sellers at some other time. It is only assumed that the switches from one pool to another tend to offset each other. ${ }^{4}$

In principle, an agent's decision on whether to trade or remain inactive is dependent on current market conditions, and may also be affected by the behaviour of other agents.

2 The same version of technical trading or trend chasing has also been employed in models by Day and Huang (1990), Brock and Hommes (1998), and Boswijk et al. (2007). In the latter two cases this becomes obvious if their price expectations are combined with the market maker scenario below.

3 Although we are concerned with daily prices and Boswijk et al. (2007) deal with annual data, it is interesting to note that their paper finds some empirical support for eqs (1) and (2). It moreover reports that the market impact of the two opposing groups is time-varying, which will actually be a key feature of our model.

${ }^{4}$ We abstain from randomizing these switches, too, as this would only blow up the notation. 
To make our point as stark as possible, we disregard such endogenous effects and treat these decisions as purely random. That is, letting $A=C, F, B, S$ stand for chartists, fundamentalists, and investors from the pool of potential buyers and sellers, respectively, each trader in group $A$ has the same probability $\pi^{A}$ of entering the market on a given day, and this probability remains fixed over time. If $N^{A}$ is the (constant) total number of agents of type $A$, the number $A_{t}$ of active traders on day $t$ of this type therefore follows a binomial distribution,

$$
A_{t} \sim \mathcal{B}\left(N^{A}, \pi^{A}\right), \quad A=C, F, B, S
$$

As a consequence, there will be an average of $\pi^{A} N^{A}$ agents in group $A$ who are active on a given market day. The variance of these activities is $\pi^{A}\left(1-\pi^{A}\right) N^{A}$, which shows that for a given number $N^{A}$ the variability in trading becomes negligible for both very small and very high probabilities $\pi^{A}$.

In general the market orders, totalling $\sum_{A} A_{t} d_{t}^{A}$, are not balanced. The excess demand (or supply) exerts a pressure on the price, causing it to move upwards (or downwards) proportionately. Introducing a positive coefficient $\mu$ to measure the impact on the price (which can be treated as a constant since the size of the population of agents was assumed to be constant), the adjustment equation reads $p_{t+1}=p_{t}+\mu \sum_{A} A_{t} d_{t}^{A}$. Using eqs (1)-(4) from above, it can be written as

$$
p_{t+1}=p_{t}+\mu\left[\left(\chi C_{t}-\phi F_{t}\right)\left(p_{t}-p^{\star}\right)+\kappa\left(B_{t}-S_{t}\right)\right]
$$

In sum, the stochastic price dynamics brought about by our model are completely described by the two equations (5) and (6).

Four points are worth pointing out. First, technically speaking the variables $C_{t}, F_{t}$, $B_{t}, S_{t}$ are just noise terms. They are, however, not imposed from the outside but are of an intrinsic nature. Conceptually, this kind of noise takes up an issue which has so far been neglected in most models that do not go into the details of the microstructure of the market. Usually the agents (or a fixed proportion of them) are supposed to trade in each time step, whereas in our eq. (5) their trading activities are - realistically, as we may claim-allowed to be variable over time. On the other hand, we do not yet seek systematic determinants of the market entry decisions but limit ourselves to a simple random mechanism. Insofar as the desired properties of the model that we will find can be attributed to effects beyond the deterministic trading rules, it is thus apparent that already these most elementary changes in the trading frequency can provide a major explanation of the stylized facts.

Second, the intrinsic noise is the only type of random perturbations that we consider, although it has to be acknowledged that this interpretation is not completely compelling. Since (under conditions that are easily satisfied here) a binomial distribution is well approximated by a Gaussian distribution, the last term in eq. (6) could also be viewed as ordinary additive and normally distributed white noise, the precise nature of which may 
not be of greater interest. The little story around the investors might then be dispensed with.

Nevertheless, as a third point we would like to emphasize that the noise originating from the fundamentalists and chartists is less customary. It is indeed linked in a multiplicative way to the gap between the actual and the fundamental price. This specification is furthermore derived from an economic reasoning and is not just a purposive technical device.

Finally, we can easily characterize the deterministic price equation that arises if the number of active traders $A_{t}$ is fixed at its average value $\pi^{A} N^{A}$ for all groups. The unique equilibrium price coincides with the fundamental value if $\pi^{B} N^{B}=\pi^{S} N^{S}$ and $\mu\left(\chi \pi^{C} N^{C}-\phi \pi^{F} N^{F}\right) \neq 0$, and global asymptotic stability prevails if and only if the latter expression is negative (but larger than -2). Since in this case (6) degenerates to a one-dimensional linear adjustment equation, the dynamic behaviour is rather uninteresting. The extreme structural poorness of the model's deterministic counterpart, which is especially due to the specification of the chartists as 'anti-fundamentalists', underscores the central role of the stochastic noise process that we have put forward.

\section{An illustrative sample run}

The model has been designed to explain - at least partially - the most important stylized facts of financial markets. ${ }^{5}$ Referring to price changes at a daily frequency, we want to check the four features that have received the most attention in the literature on agentbased models. These are the absence of autocorrelations in the raw returns, fat tails in their frequency distributions, volatility clustering, and long memory (see Chen et al., 2009, p. 19), and we will consider them in both qualitative and quantitative terms. With a view to stock markets, which will be discussed shortly, we settle on the numerical parameters given in Table 1.

\begin{tabular}{ccccccccc}
$\chi$ & $\phi$ & $\kappa$ & $\mu$ & $p^{\star}$ & $N^{C}=N^{F}$ & $\pi^{C}=\pi^{F}$ & $N^{B}=N^{S}$ & $\pi^{B}=\pi^{S}$ \\
\hline 1.000 & 1.011 & 0.165 & 0.010 & 0.00 & 200 & 0.50 & 1000 & 0.010
\end{tabular}

Table 1: The stock market parameter scenario.

As can be seen, we assume an equal number of chartists and fundamentalists who on average trade every second day, and an equal, considerably larger number of buying and selling investors who on average only trade every 100 days. In particular, the equilibrium

${ }^{5}$ Detailed descriptions of the statistical properties of asset prices can be found in Cont (2000), Lux and Ausloos (2002), or Lux (2009b). 
price is thus equal to the fundamental value $p^{\star}$, the $(\log )$ level of which is arbitrary and has been normalized at zero for concreteness. The coefficient $\mu$ can be used to scale the parameters $\chi, \phi, \kappa$ and has been set such that the responsiveness $\chi$ of the chartists equals unity. The fundamentalists are assumed to be slightly more aggressive, so that the expected value of the random coefficient $\chi C_{t}-\phi F_{t}$ on the price is negative in (6) and the dynamics generated by these two groups is mean-reverting in the long run (without overshooting the fundamental value, since $1-\mu\left(\chi \pi^{C} N^{C}-\phi \pi^{F} N^{F}\right)$ is positive). As already indicated at the end of the previous section, the term $\mu \kappa\left(B_{t}-S_{t}\right)$ in (6) could be regarded as additive white noise, the variance of which is given by the product of $\mu^{2} \kappa^{2}$ and $\pi^{B}\left(1-\pi^{B}\right) N^{B}+\pi^{S}\left(1-\pi^{S}\right) N^{S}$.

The dynamic process governing the market price can therefore be summarized as an interplay of two stochastic laws, one of which is additive and the other multiplicative. Without the investors, the price would eventually converge toward the fundamental value. That is, $p^{\star}$ would be approached under random fluctuations that become increasingly smaller over time. Here it is the very role of the investors to permanently offset this tendency. On the other hand, without the chartists and fundamentalists the process would degenerate to an ordinary random walk. Their presence and the slight long-run dominance of the stabilizing fundamentalists over the chartists prevents the price from diverging too far from its equilibrium or, more technically, ensures that its variance remains bounded. Given the number of agents and their trading probabilities, the overall noise level of the process is determined by the trading volume $\kappa$ of the active investors, and its value in Table 1 will turn out to be of a suitable order of magnitude.

Figure 1 presents a sample run of the model with the parameters from Table 1 . It is computed over the same time span as the daily data of the S\&P 500, to which it will be compared. The series thus covers 6767 days, which are 100 days less than the period for the S\&P 500 from January 1980 to mid-March $2007 .{ }^{6}$

The upper panel illustrates the fluctuations of the price around the fundamental value $p^{\star}=0$, revealing that they occur in an irregular manner. There are indeed longer periods of tranquillity where the price remains close to $p^{\star}$, but they are occasionally disrupted by larger bubbles where the price disconnects from $p^{\star}$. From the description above it is clear that the bubble episodes can result from a temporary prevalence of the random walk tendencies in the price process when the chartists and fundamentalists largely neutralize each other. An alternative, or additional, reason is a (likewise temporary) predominance of the chartists over the fundamentalists, which causes a monotonic divergent motion of the price.

Larger deviations of the price from the fundamental value will also affect volatility. When either the chartists or fundamentalists become dominant for a few days within the

6 This means that the entire series covers 6867 days, from which the first 100 days are set aside to allow the computation of autocorrelations up to a lag of 100 days from $t=1$ onward. 

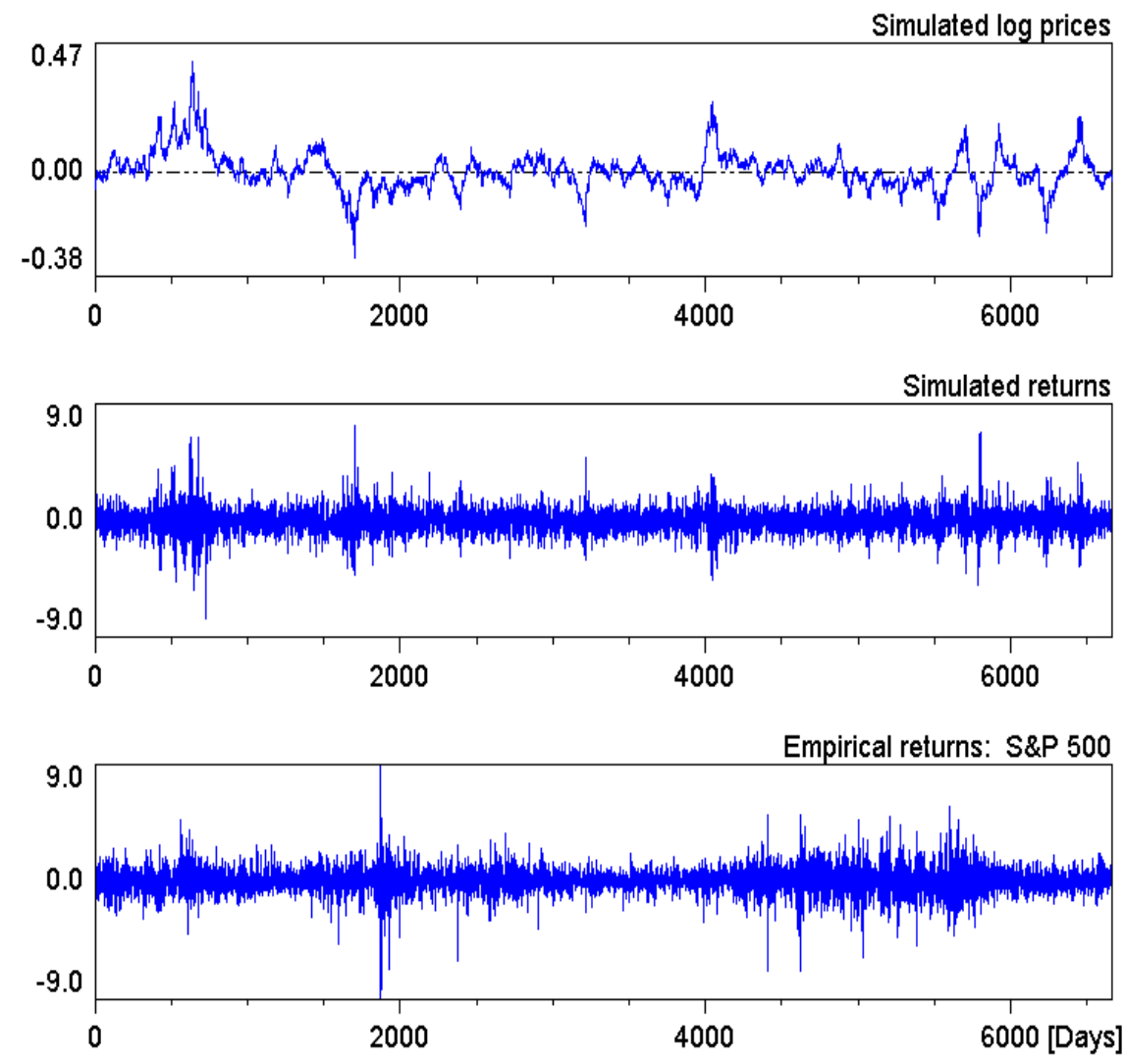

Figure 1: Simulated and empirical time series.

group of the speculators in such a situation, and the buying and selling investors more or less offset each other, the combination of the multiplicative noise and the larger gap between $p_{t}$ and $p^{\star}$ leads to price changes that are well above normal. In other words, the occasional misalignment in the price causes a volatility clustering in the returns, or at least it increases the likelihood of particularly high or low returns. This is clearly visible in the middle panel of Figure 1; typical examples are the periods around $t=600, t=1700$, or $t=5750$. We may also anticipate from this phenomenon that the distributions of the returns will not be normal.

The bottom panel in the diagram displays the returns from the S\&P 500 over the same time horizon. A comparison with the middle panel shows that the pattern of the alternation of periods of tranquillity and volatility in the returns is similar for the simulated and empirical series. Also the quantitative outbursts are comparable in size (note that the second and third panels have the same scale). Differences can be seen in the band width of the returns in the periods of relative tranquillity. While the noise level is then constant in the simulated series, the empirical series exhibit certain changes from the first, say, 1800 days to the period between $t=3000$ and $t=4000$, where the band 
becomes narrower, and from there to the period between $t=4700$ and $t=5700$, which seems to be almost on the edge of tranquillity. Clearly, a simple model cannot account for such 'regime shifts' (if they were found to be significant at all).

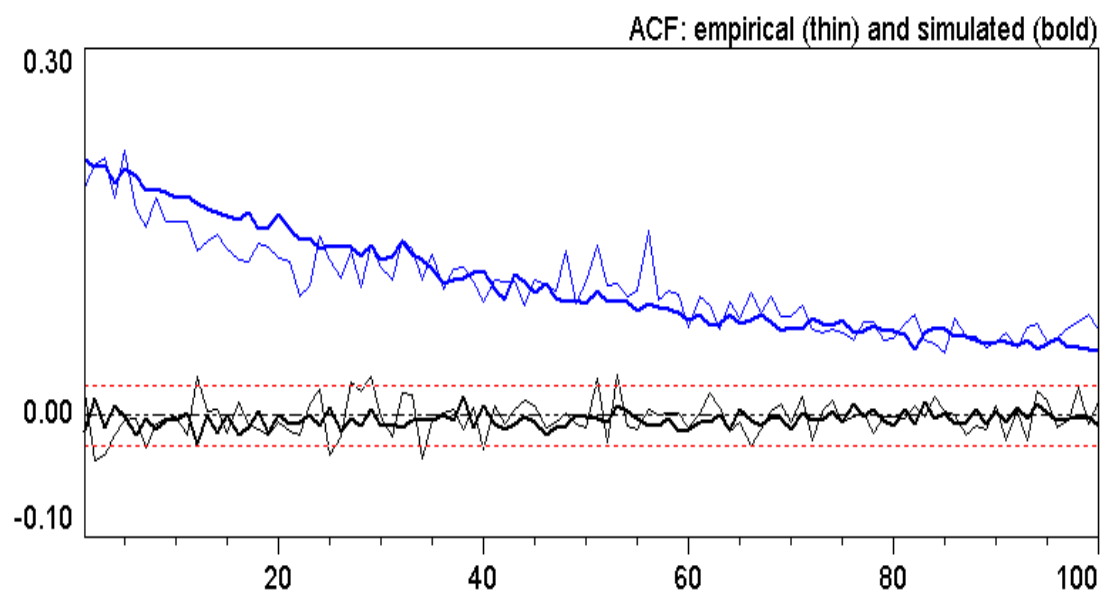

Figure 2: Autocorrelation functions of returns.

Note: Thin (bold) lines indicate empirical (simulated) returns, the two upper (lower) lines represent the ACF of absolute (raw) returns, and the dotted lines mark the insignificance band (at the 95\% level). The underlying simulation horizon is 10 times the empirical horizon, i.e., 67,670 days.

The time scale of Figure 1 does not allow us to assess the behaviour of the returns over a few consecutive days. As the model is set up, however, we have every reason to believe that it can reproduce the stylized fact of uncorrelated returns. This is confirmed for lags from 1 to 100 days by the lower bold line in Figure 2. To obtain a smoother shape of the autocorrelation function $(\mathrm{ACF})$, the coefficients are based on a 10 times longer simulation horizon of 67,670 days. ${ }^{7}$ For this larger sample none of the coefficients is significantly different from zero. Likewise, as shown by the lower thin line, they are not significantly different from their empirical counterparts.

The other two lines in the diagram take us back to the issue of volatility clustering. Note that this concept, which describes the tendency of large changes in the asset price to be followed by large changes and small changes to be followed by small changes, is closely related to the long-term dependencies between returns, a phenomenon usually referred to as long memory. A common quantitative characteristic of these effects is the $\mathrm{ACF}$ of squared or absolute returns. Since one is as good as the other, Figure 2 plots the autocorrelation coefficients of the first 100 lags of the absolute returns. These statistics

7 Since it reduces the overall computational effort by roughly two-thirds, this and the simulations to follow have approximated the binomial deviates with random draws from the corresponding normal distribution; see Press et al. (1986, Section 7.3) for the computational background. 
are a crucial test for a model's ability to reproduce the empirical long memory effects, and the diagram documents that the present model passes this test with success.

As an additional feature, we report our evidence of the non-normality of the returns. Measuring the fatness of the tail by the well-known Hill estimator, where we specify the tail as the upper 5 per cent of the absolute returns, practically no difference is found between the simulated and empirical series: the Hill estimator $\hat{\alpha}_{H}$ of the latter is 3.30, while our (extended) sample run yields $\hat{\alpha}_{H}=3.27$.

The section should nevertheless be concluded with a word of caution. Although the results for the ACF of the raw and absolute returns as well as the Hill estimator may seem highly gratifying, the model is still rejected by harder econometric criteria that compile this information in a single objective function. A suitable tool in this respect, which has the advantage of being more transparent than other simulation-based econometric procedures, is the method of simulated moments (see Lee and Ingram, 1991, or Duffie and Singleton, 1993, as standard references; or Franke, 2008a, for a recent application). Without going into detail, let us here briefly mention that in our application of this approach we employed nine moments: one for the variance of the raw returns $r_{t}$, one for the mean of the absolute returns, one for the first-order autocovariance of $r_{t}$, four for the autocovariances of $\left|r_{t}\right|$ at different short and long lags, and two moments reflecting the Hill estimator. The econometric theory rejects a model if the objective function $J$ that is built up from these moments exceeds a certain value of the $\chi^{2}$-distribution. The critical value depends on the degrees of freedom involved (which in turn depend on the number of free parameters that we are trying to estimate) and is here typically in the region of 10 or 12 . By contrast, we obtain $J \approx 50$ for our simulation run over the long horizon. This econometric result puts the above findings into perspective and, in particular, prevents us from selling our model as a "true" model. However, the matching properties that have been obtained so far in Figures 1 and 2 and in the computation of the tail index can still be regarded as fairly satisfactory, or at least competitive.

\section{A more systematic investigation}

Even if the results presented in the previous section may be attractive, it should not be forgotten that they were based on a single simulation run-which we might have been accordingly selected. As a matter of fact, when repeating the experiment with other random number sequences we can also observe quite different summary statistics, despite the seemingly comfortably long sample period of over 67,000 time steps. The problem of the model's robustness therefore has to be taken seriously.

We undertook a Monte Carlo study to treat the problem in a systematic way. To this end, 5000 simulations were run with different random seeds over 67,670 days. Before turning to the results themselves it should be mentioned that in rare cases the price might wander so far away from the fundamental value that the randomly occurring 
larger differences in the trading activities of fundamentalists and chartists lead to an extreme excess demand for the asset. The returns over these episodes, which at most last a few hundred days, are occasionally so disproportionately high or low that the entire summary statistics are completely out of line. The phenomenon could easily be prevented by introducing a suitable nonlinearity in the formulation of demand. For example, it would be quite reasonable to assume that beyond a certain threshold of mispricing, the confidence of the fundamentalists in their trading strategy rises relative to the confidence of the chartists. In other words, the trading probabilities of the fundamentalists increase and those of the chartists decrease, thus reinforcing the mean reversion tendencies on the market. The overly strong bubbles, however, are so exceptional that such a remedy does not appear worthwhile.

Accordingly, if such an event was encountered in a simulation run, we simply discarded the entire sample. ${ }^{8}$ This occurred in 360 out of 5360 simulation runs. If it is (very conservatively) assumed that the extreme bubbles last no longer than 500 days, we would have an upper-bound of $360 \cdot 500=180,000$ days of excessive volatility out of a total of $67,670 \cdot 5360 \approx 3.62$ billion days. Hence this rough-and-ready assessment says the probability that the unadjusted model produces a (hitherto) unrealistic bubble is less than $0.049 \%$. We believe this is a reasonable figure to justify what has just been called "not worthwhile".

This being understood, we can compute a set of summary statistics for each simulation run and thereby obtain a frequency distribution of them on the basis of 5000 samples. Let us begin with a measure for volatility, which we define here as the standard deviation of the raw returns. Given that this magnitude is basically a matter of scaling the model and that each simulation runs extends over 271 years, a lower sample variability of the model may have been expected than the range of 0.93 for the $2.5 \%$ quantile and 1.06 for the $97.5 \%$ quantile. ${ }^{9}$ On the other hand, the median with a value of 0.98 is not very different from the empirical volatility of 1.02 . The distribution of the Hill estimator may not appear too narrow, either: with roughly 3 and 4 as its lower and upper boundary, respectively, the $95 \%$ confidence interval covers the whole range of what has been estimated for a large number of stock markets. In any case, the empirical Hill estimator of the S\&P 500 is well within that interval.

The density functions of the volatility and the Hill estimator from the Monte Carlo experiment are plotted in the upper two panels of Figure $3 .{ }^{10}$ Higher values of the trading capital $\kappa$ could shift the volatility distribution in the top-left panel further to the right, so

8 To be precise, the event was specified by $J>150$ for the objective function outlined above.

9 These numbers and the other statistics discussed here are documented in Table A1 in the appendix. In addition, the table reports two (representative) autocorrelation coefficients for the raw returns to confirm their overall insignificance.

${ }^{10}$ The density functions have been estimated by means of the Epanechnikov kernel; see Davidson and MacKinnon (2004, pp.678-683). 

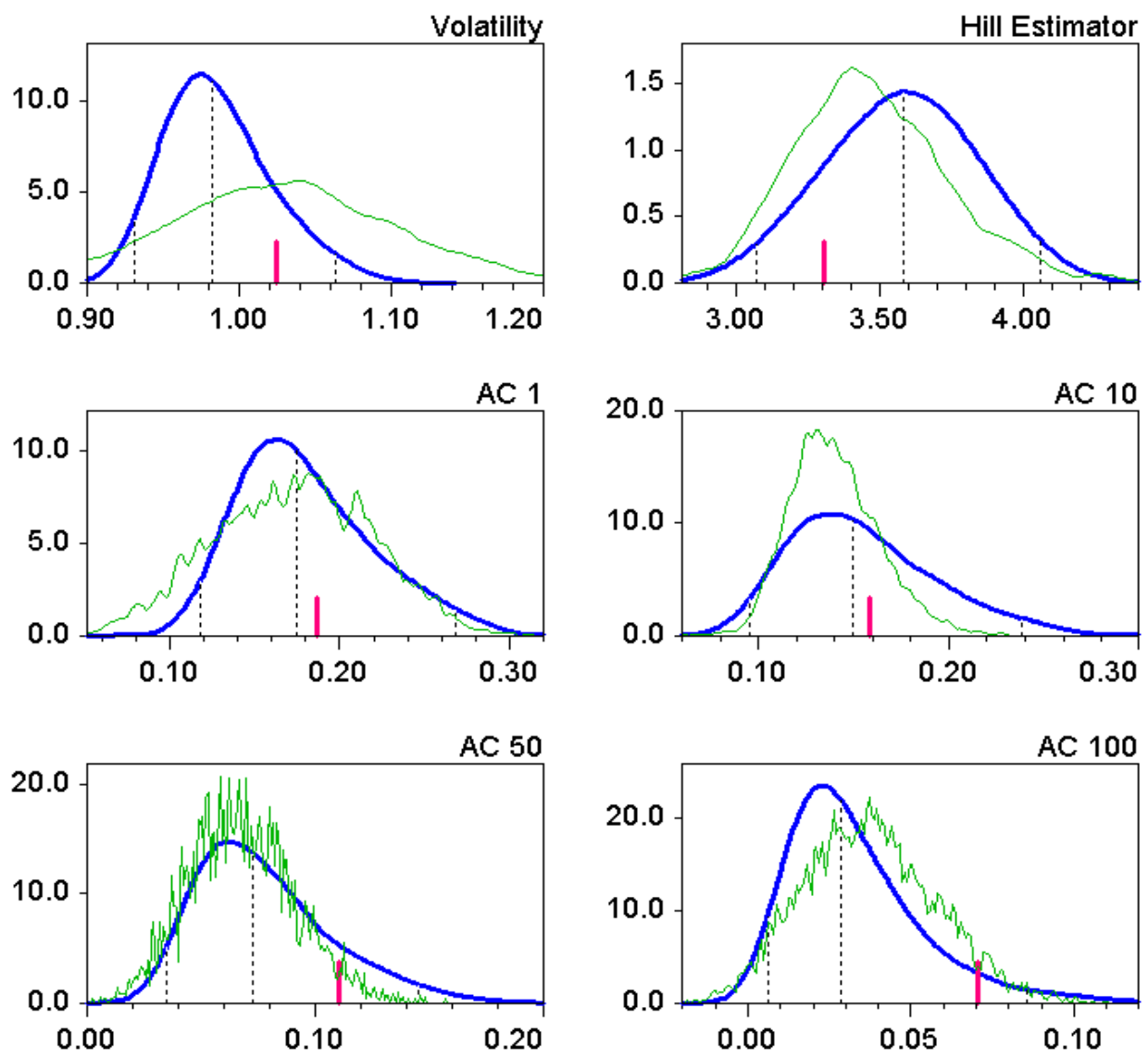

Figure 3: Frequency distributions of selected summary statistics.

Note: The bold (blue) line plots the frequency distributions generated by the parameter values in Table 1; they are based on 5000 simulation runs over 10 times the empirical horizon. The vertical dotted lines indicate their $2.5 \%$ quantile, the median and the $97.5 \%$ quantile, respectively, while the bold (red) bar indicates the empirical summary statistic. The thin solid (green) line represents the bootstrapped frequency distributions, which are likewise based on 5000 samples.

that the median would be closer to the empirical volatility. Fixing the other parameters there are, however, limits to this procedure since then some of the other statistics would begin to deteriorate (the overall outcome of which we could evaluate by the objective function $J$ that was briefly outlined at the end of the last section).

The other four panels in Figure 3 demonstrate the density functions of the autocorrelation coefficients of the absolute returns at lags 1, 10, 50 and 100. Again, in all four cases the empirical statistic is well within the $95 \%$ confidence interval, and for the lags 1 and 10 it is pleasantly close to the median of the Monte Carlo samples. Nevertheless, the support of the distributions also seems rather wide again, where at the longer lags a small but non-negligible part of the samples even yields insignificant coefficients near zero. 
On the whole, the distributions in Figure 3 may convey the impression of a limited robustness of the model. Such an evaluation needs, however, to be put into perspective. For this reason we ask what kind of variation in the statistics could be expected from the empirical data itself. This question can be answered by a bootstrap procedure or, more precisely, due to the serial correlation in the absolute returns, by a block bootstrap. A suitable block length is a year, i.e. 250 days. Accordingly we reduce the empirical return series of the S\&P 500 to 6750 observations, subdivide it into 27 blocks of 250 days, and construct a new series block by block from 27 random draws (with replacement), from which all of the desired statistics can then be computed. Repeating this 5000 times, we have an equally large sample of bootstrapped statistics available and can draw the density functions for them. These are the thin solid (green) lines in Figure 3.

The important message from this check is that by and large the bootstrap distributions are similarly wide to the distributions of the statistics from the Monte Carlo exercise. ${ }^{11}$ Hence the widths of the model-generated frequency distributions are quite in line with what we can, or even should, expect from the repeated simulations of a model.

Before the "fit" of the model's frequency distributions to the bootstrap distributions in Figure 1 is seen too positively, it should be recalled that these are only the marginal distributions of the single statistics and that the coincidence in the joint distributions could be much weaker. In this respect we should come back to the objective function $J$ already mentioned, which characterizes the goodness-of-fit of the empirical summary statistics, or moments, by a single number. Computing the frequency distributions of the values of $J$ for both the bootstrap and the model-generated series, we practically find no overlap between the two distributions. This discrepancy is so pronounced that again there is no scope for mistaking the model as a "true" model. Again, we may evaluate the model's ability to mimic the stylized facts of interest to us as "satisfactory, though not perfect".

\section{Validating the model for a foreign exchange market}

We need not make a statement in this paper about whether econometric methods applied to the daily returns are indeed capable of telling apart the major stock indices of the world. In any case, the broad confidence intervals in the Monte Carlo frequency distributions in Figure 3 have demonstrated that this would probably demand too much from the present model. On the other hand, visual inspection of, in particular, the ACF

\footnotetext{
${ }^{11}$ The much wider bootstrap distribution of the volatility could be explained by the different noise levels of the S\&P 500 returns over longer periods of time, which we have already touched on in the discussion of Figure 1. Normally there are multiple random draws of the same block at the cost of other blocks that do not appear at all in the bootstrap series. This causes a certain dispersion of the volatility measure across the 5000 samples. In contrast, as has also been noted in the discussion of Figure 1, the model's noise over the (relatively) tranquil periods is more homogeneous.
} 
of the absolute returns is sufficient to distinguish between the leading stock and foreign exchange (FX) markets. We may therefore ask how far we can get with our model in this respect. Our empirical series for this investigation will be the USD-DEM exchange rate over the same time horizon as before, from January 1980 to mid-March 2007.

Even a full-fledged estimation procedure - if it made sense after all - could not possibly identify all of the parameters of the model. Besides the fundamental value $p^{\star}$, of course, we chose to fix the market impact coefficient $\mu$, the reaction coefficients $\chi$ and $\phi$ of the chartists and fundamentalists, and the trading capital $\kappa$ and trading probability $\pi^{B}=\pi^{S}$ of the buying and selling investors. A readjustment was undertaken for the number $N^{B}=N^{S}$ of the two types of investors, the number $N^{C}=N^{F}$ of the two types of speculators, and the trading probability $\pi^{C}=\pi^{F}$ of the latter. Table 2 shows the values on which we now agree.

\begin{tabular}{ccccccccc}
$\chi$ & $\phi$ & $\kappa$ & $\mu$ & $p^{\star}$ & $N^{C}=N^{F}$ & $\pi^{C}=\pi^{F}$ & $N^{B}=N^{S}$ & $\pi^{B}=\pi^{S}$ \\
\hline 1.000 & 1.011 & 0.165 & 0.010 & 0.00 & 275 & 0.60 & 525 & 0.010
\end{tabular}

Table 2: The FX market parameter scenario.

Comparing the table to the stock market parameter scenario in Table 1, it can be seen that the FX scenario assigns a much weaker role to the long-term investors (525 versus 1000), and a stronger role to the speculators concerning both their absolute number (275 versus 200 ) and their trading frequency (0.60 versus 0.50 ). Applying the method of simulated moments indicated above, we also treated this as an estimation problem. According to the econometric standards provided by the method, the changes in $N^{B}=$ $N^{S}$ and $\pi^{C}=\pi^{F}$ (but not $N^{C}=N^{F}$ ) are highly significant. Without carrying the analogy too far, it could be said that the signs of the modifications from Table 1 to Table 2 correspond to the general verbal characterizations of stock and FX markets. If, on the whole, the fitting properties of the two parameter scenarios are deemed acceptable, the model could thus be said to have some explanatory power. ${ }^{12}$

To see the consequences of the parameter changes, we first simulate the model with the same random number sequence as in Section 3. The results are presented in Figure 4. A comparison of the top panel of this figure with that in Figure 1 shows that the pattern of the evolution of the price is almost perfectly maintained. The only essential

\footnotetext{
${ }^{12}$ While there are far more traders on the FX than on the stock markets, when considering the absolute number of agents in the model the ceteris paribus condition of the fixed market impact coefficient $\mu$ should be taken into account. A more scrupulous modelling approach might scale it in some way with the overall number of agents, but the problem of how precisely this should be specified seems to be terra incognita in all models where the market prices are assumed to respond to total excess demand (since the number of traders is usually fixed).
} 

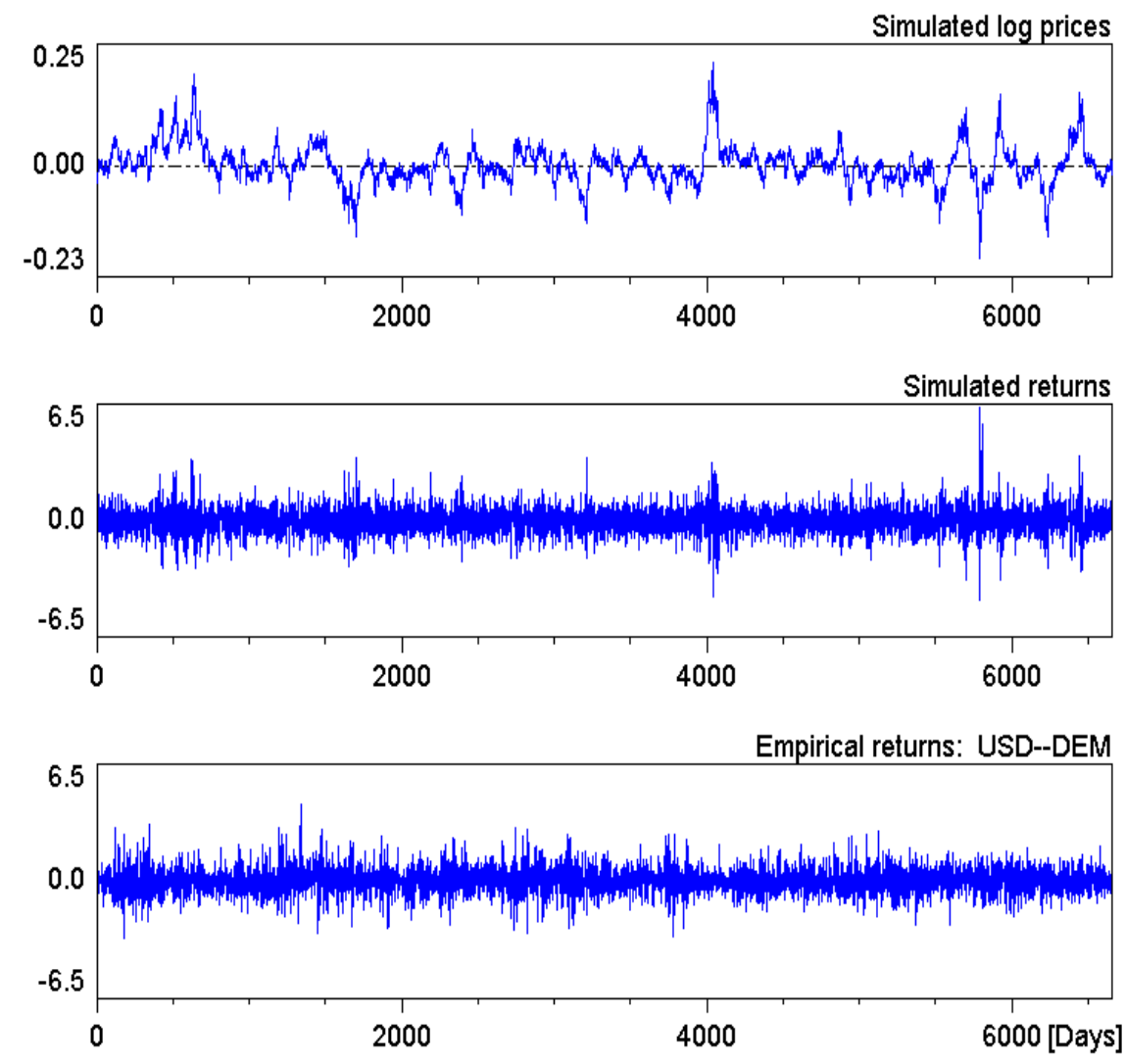

Figure 4: Simulated and empirical time series (FX market scenario).

difference is a reduction in size. The reason for this phenomenon is obvious: the model's random walk tendencies are weaker owing to the lower level of the additive noise, which is caused by the fewer investors.

At first glance, the higher weight of the chartists and fundamentalists on the market might be suggestive of an increase in the volatility of returns. The noise originating from this side, however, is multiplicative. That is, the random term $\left(\chi C_{t}-\phi F_{t}\right)$ in the price equation (6) is multiplied by the price gap $\left(p_{t}-p^{\star}\right)$. Although in the new scenario the first term increases in modulus, the price gaps have been seen to decline. In fact, the second effect turns out to be dominant. As can be seen from a comparison of the simulated returns in Figures 1 and 4, the price changes in the bubble periods are now considerably smaller (notice also the lower scale of the return panels in Figure 4). The return series still exhibits a certain degree of volatility clustering, but it is clearly less pronounced than in the stock market scenario.

Lower fluctuations of the returns, on average as well as in their more extreme values, are also observed in the return series of the USD-DEM exchange rate; see the bottom panel of Figure 4. On the basis of a mere visual inspection, one might even wonder if volatility 
clustering is present here at all. Regarding its numerical characterization, at least the corresponding long memory effects should be distinctly weaker. This is clearly brought out by the upper thin line in Figure 5, which plots the ACF of the absolute returns of the empirical exchange rate. Nevertheless, over the first 50 lags the long memory effects have not completely disappeared, either.

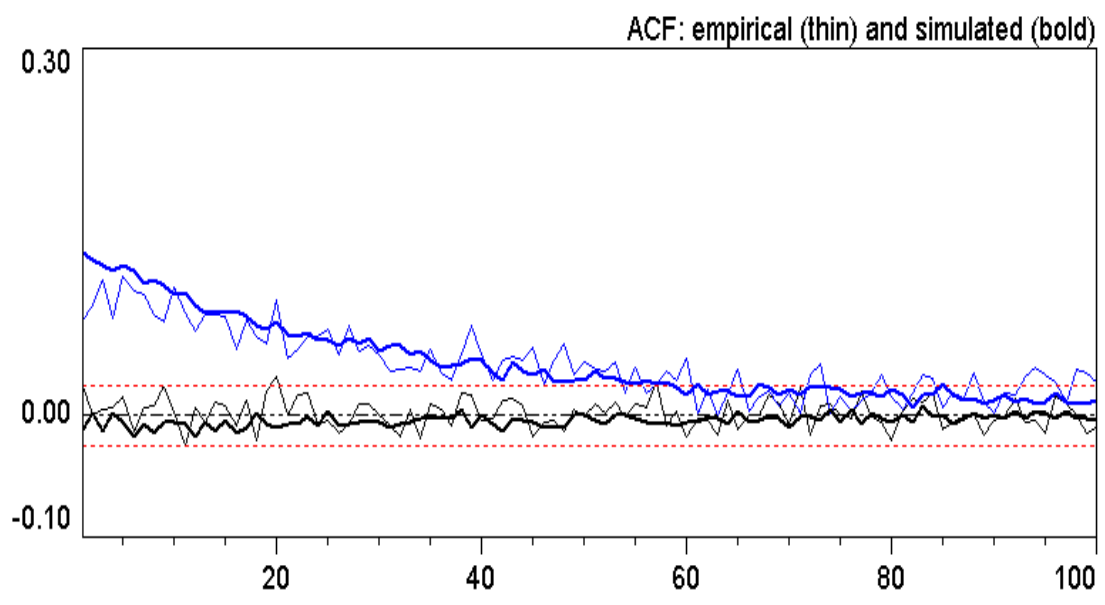

Figure 5: Autocorrelation functions of returns (FX market scenario).

Note: See legend in Figure 2; the underlying empirical series here is the USD-DEM exchange rate.

The diagram above also demonstrates that the model-generated ACF of the absolute returns can match its empirical counterpart for most of the lags. Only the first few lags are somewhat overestimated. The problem is that while the shape of the ACFs produced by the model typically comes close to a kind of power law, which is so attractive in the theory of financial markets, the empirical ACF might be too flat over the first few lags for such a stylized description. Correspondingly, if another choice of the model parameters lowers the bold line over an initial lag interval, this ACF would tend to be below the empirical thin line at the longer lags. The "fitting" in Figure 5 is a compromise in this respect. Nonetheless, the serial correlation in the raw returns is again insignificant for both the empirical and artificial series.

To detach ourselves from this example of a selected simulation run, we conduct the same battery of experiments as in Section 4 for the stock market scenario, resulting in Figure 6 as a counterpart to Figure 3 . The bold lines represent the frequency distribution of the 5000 simulations of the model, and the thin solid lines to which they are contrasted are the frequency distributions of the 5000 (block) bootstrap samples of the returns from the empirical exchange rate. The two distributions are fairly congruent for the volatility, the Hill estimator and the autocorrelations at lags 10 and 50. The fact that the empirical coefficient at lag 100 exceeds the $97.5 \%$ quantile of the model-generated distribution is 

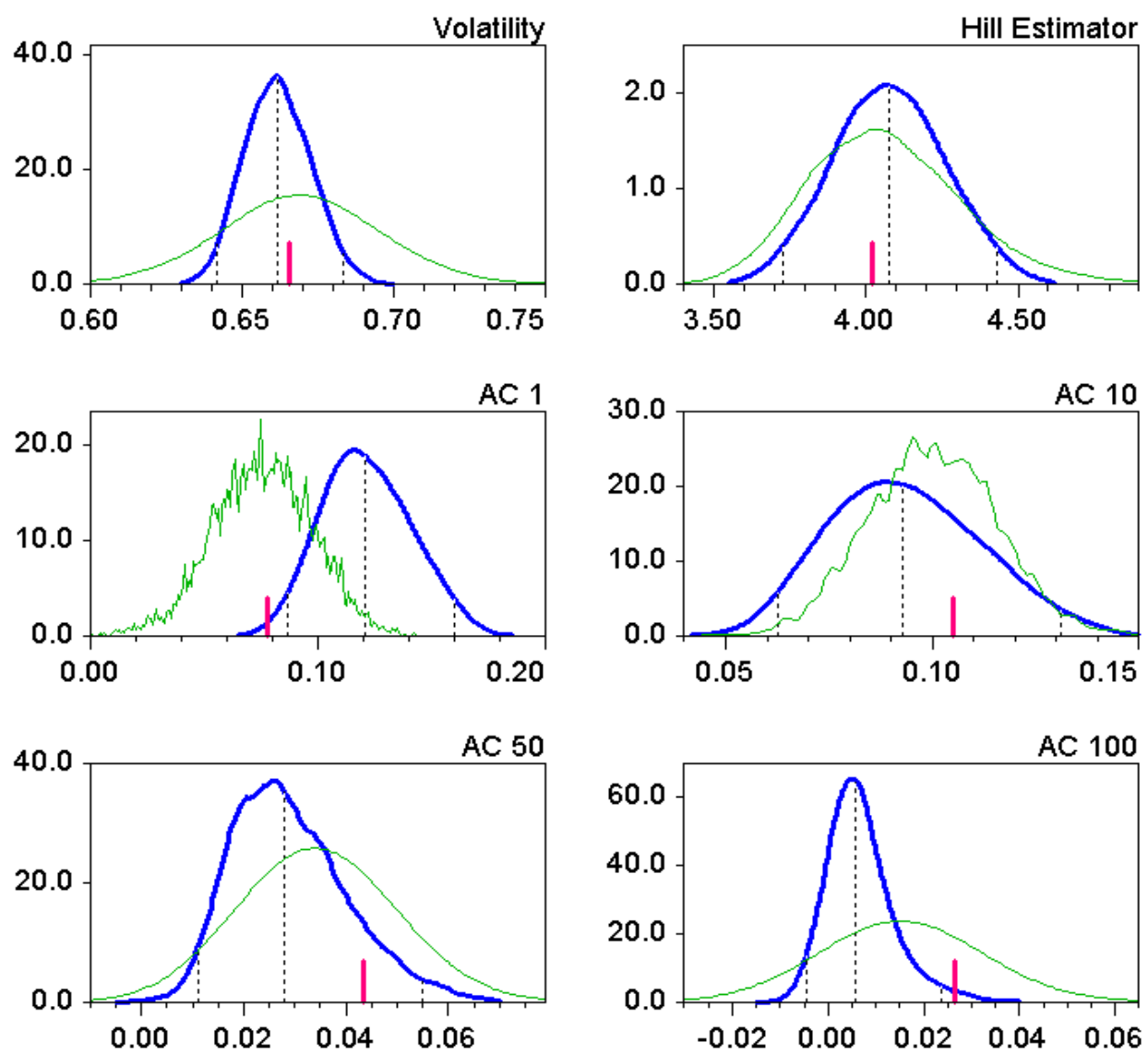

Figure 6: Frequency distributions of selected summary statistics (FX market scenario).

Note: See legend in Figure 3; the underlying empirical series here is the USD-DEM exchange rate.

not very dramatic since its bootstrap distribution centres more to the left and larger parts of the two distributions are in a region of insignificance anyway.

On the other hand, the overestimation of the first-order autocorrelation of the absolute returns in Figure 5 was no exception. The two middle panels in Figure 6 show that the model's distribution of this coefficient covers a higher range than the distribution of the lag-10 coefficients, whereas the opposite is true for the bootstrap distributions of the empirical returns (see Table A2 in the Appendix for the precise quantiles). Accepting this limitation, however, we can conclude that the model's moment matching is still of a satisfactory quality, and that the alternative parameter scenario in Table 2 provides a respectable explanation of the different features of the return series from stock and foreign exchange markets. 


\section{Conclusion}

The paper has proposed a minimal structural model that introduces intrinsic stochastic noise in the form of a probabilistic market participation of the individual agents. Applying this noise to the fundamentalist and chartist strategies, we thus obtain a source of randomness that is multiplicatively linked to the price. Applying the intrinsic noise to our type of investors, who randomly buy and sell the asset, an additive random mechanism emerges. Its main role is to prevent the price dynamics (or the multiplicative noise, so to speak) from dying out. Finally, fundamentalists and chartists were specified as trading in opposite directions. As a result, the price dynamics will occasionally become unstable and tend to diverge from the fundamental value. Since, on the other hand, fundamentalism was supposed to have a weak dominance in the long run, the price will eventually return to its benchmark. In short, periods of tranquillity and a certain turmoil alternate in an unpredictable though balanced fashion, which is the core of the phenomenon of volatility clustering.

Our study also went beyond such a qualitative description and attempted to match a number of summary statistics of the empirical returns from the S\&P 500 or, alternatively, the USD-DEM exchange rate, which besides the absence of serial correlation in the raw returns reflect the fat tails and the long memory effects in the autocorrelation pattern of the absolute returns. Although the fit is not perfect, given the extreme parsimony of the model we nevertheless consider it to be fairly satisfactory. In this sense, the model can be claimed to be "validated". We would also like to add that it is still a challenge to other structural small-scale models to catch up with the present standard of "moment matching". 13

A possible criticism of our model may be directed against the converse trading strategies of fundamentalists and chartists. A simple specification of the trend-following behaviour of technical traders along the lines of, say, Beja and Goldman (1980), or a movingaverage rule as in Chiarella et al. (2006), may be a straightforward and attractive idea here. Our conjecture is that since the fundamentalist and chartist strategies would then also act against each other in the price dynamics, albeit with a short time delay, the basic mechanism generating the volatility clustering would be essentially maintained. The advantage of the converse strategies is that they make the mechanism even more transparent, which is why we adhered to them.

Generally, we believe that the present model and the results we obtained from it are not only interesting per se but could also stimulate the design of other small-scale models.

\footnotetext{
${ }^{13}$ According to the explicit method-of-simulated-moments estimation in Franke (2008a), the threshold switching model by Manzan and Westerhoff (2005) appears to be somewhat superior to the present model. Franke (2008b) discusses two other, structurally very appealing models from the literature, which reveals that they cannot reasonably keep their promises of matching the stylized facts.
} 
A promising avenue for future research might be the endogeneization of the individual probabilistic trading frequencies which, with regard to their expected values, might correspond to the strategy switching mechanisms introduced by Lux (1995) or Brock and Hommes (1998). Considering the relatively strong contribution of our highly parsimonious model to the stylized facts, we would be interested in learning which minimal extensions in these directions would lead here to a significant improvement, which is still desirable.

\section{References}

Beja, A. and Goldman, M.B. (1980): On the dynamic behavior of prices in disequilibrium, Journal of Finance, 35, 235-248.

Boswijk, P., Hommes, C. and Manzan, S. (2007): Behavioral heterogeneity in stock prices. Journal of Economic Dynamics and Control, 31, 1938-1970.

Brock, W. And Hommes, C. (1998): Heterogeneous beliefs and routes to chaos in a simple asset pricing model. Journal of Economic Dynamics Control, 22, 1235-1274.

Brock, W. And Hommes, C. (1998): Heterogeneous beliefs and routes to chaos in a simple asset pricing model. Journal of Economic Dynamics Control, 22, 1235-1274.

Chen, S.-H., Chang, C.-L. And Du, Y.-R. (2008): Agent-based economic models and econometrics. Paper presented at the Econophysics Colloquium, Kiel, August 2008.

Chiarella, C., Dieci, R. And He, X.-Z. (2009): Heterogeneity, market mechanisms, and asset price dynamics. In: Hens, T. and Schenk-Hoppé, K.R. (eds): Handbook of Financial Markets: Dynamics and Evolution. North-Holland: Amsterdam; pp. 277-344.

Chiarella, C., He, X.-Z. And Hommes, C. (2006): A dynamic analysis of moving average rules. Journal of Economic Dynamics and Control, 30, 1729-53.

Cont, R. (2001): Empirical properties of asset returns: stylized facts and statistical issues. Quantitative Finance, 1, 223-236.

Davidson, R. And MacKinnon, J.G. (2004): Econometric Theory and Methods. Oxford University Press: Oxford.

Day, R. And Huang, W. (1990): Bulls, bears and market sheep. Journal of Economic Behavior and Organization, 14, 299-329.

Duffie, D. And Singleton, K.J. (1993): Simulated moments estimation of Markov models of asset prices. Econometrica, 61, 929-952.

Franke, R. (2008a): Applying the method of simulated moments to estimate a small agentbased asset pricing model. Working Paper, University of Kiel (www.bwl.uni-kiel.de/gwif/downloads_papers.php?lang=en).

Franke, R. (2008b): Artificial long memory effects in two agent-based asset pricing models. Working Paper, University of Kiel (www.bwl.uni-kiel.de/gwif/downloads_papers.php?lang=en).

Graham, B. And Dodd, D. (1951): Security Analysis. McGraw Hill: New York.

Hommes, C. (2006): Heterogeneous agent models in economics and finance. In: Tesfatsion, L. and Judd, K. (eds): Handbook of Computational Economics, Vol. 2: Agent-Based Computational 
Economics. North-Holland: Amsterdam; pp. 1107-1186.

Hommes, C. And Wagener, F. (2009): Complex evolutionary systems in behavioral finance. In: Hens, T. and Schenk-Hoppé, K.R. (eds): Handbook of Financial Markets: Dynamics and Evolution. North-Holland: Amsterdam; pp. 217-276.

KIRman, A. (1991): Epidemics of opinion and speculative bubbles in financial markets. In: Taylor, M. (ed.): Money and Financial Markets. Blackwell: Oxford; pp. 354-368.

LeBaron, B. (2006): Agent-based computational finance. In: Tesfatsion, L. and Judd, K. (eds): Handbook of Computational Economics, Vol. 2: Agent-Based Computational Economics. NorthHolland: Amsterdam; pp. 1187-1233.

LeE, B.-S. And Ingram, B.F. (1991): Simulation estimation of time series models. Journal of Econometrics, 47, 197-205.

Lux, T. (1995): Herd behaviour, bubbles and crashes. Economic Journal, 105, 881-896.

Lux, T. (2009a): Applications of statistical physics in finance and economics. In: Rosser, J.B., Jr. (ed.): Handbook of Research on Complexity. Edward Elgar: Cheltenham (in press).

Lux, T. (2009b): Stochastic behavioural asset-pricing models and the stylized facts. In: Hens, T. and Schenk-Hoppé, K.R. (eds.): Handbook of Financial Markets: Dynamics and Evolution. North-Holland: Amsterdam; pp. 161-216.

Lux, T. AND Ausloos, M. (2002): Market fluctuations I: Scaling, multiscaling, and their possible origins. In: Bunde, A., Kropp, J. and Schellnhuber, H. (eds): Science of Disaster: Climate Disruptions, Heart Attacks, and Market Crashes. Springer: Berlin; pp. 373-410.

Lux, T. AND MARChESI, M. (1999): Scaling and criticality in a stochastic multi-agent model of a financial market. Nature, 397, 498-500.

Manzan, S. and Westerhoff, F. (2005): Representativeness of news and exchange rate dynamics. Journal of Economic Dynamics and Control, 29, 677-689.

Menkhoff, L. And TAYLOR, M. (2007): The obstinate passion of foreign exchange professionals: technical analysis. Journal of Economic Literature, 45, 936-972.

Murphy, J. (1999): Technical Analysis of Financial Markets. New York Institute of Finance: New York.

Press, W.H. et AL. (1986): Numerical Recipes: The Art of Scientific Computing. Cambridge University Press: Cambridge, UK.

Westerhoff, F. (2009): Exchange rate dynamics: A nonlinear survey. In: Rosser, J.B., Jr. (ed.): Handbook of Research on Complexity. Edward Elgar: Cheltenham (in press).

Westerhoff, F. And Dieci, R. (2006): The effectiveness of Keynes-Tobin transaction taxes when heterogeneous agents can trade in different markets: A behavioral finance approach. Journal of Economic Dynamics and Control, 30, 293-322. 


\section{Appendix:}

\section{Summary statistics of the Monte Carlo and bootstrap experiments}

\begin{tabular}{|c|c|c|c|c|c|c|c|c|}
\hline \multirow[b]{2}{*}{$Q$} & \multirow[b]{2}{*}{$V$} & \multirow[b]{2}{*}{$H$} & \multicolumn{6}{|c|}{ Autocorrelations ( of $r_{t} \mid$ of $\left|r_{t}\right|$ ) at Lag } \\
\hline & & & 1 & 10 & 1 & 10 & 50 & 100 \\
\hline \multirow[t]{2}{*}{$2.5 \%$} & 0.93 & 3.07 & -0.020 & -0.019 & 0.118 & 0.096 & 0.035 & 0.006 \\
\hline & 0.88 & 3.00 & -0.014 & -0.029 & 0.082 & 0.101 & 0.026 & 0.000 \\
\hline \multirow[t]{2}{*}{$50.0 \%$} & 0.98 & 3.58 & -0.006 & -0.005 & 0.174 & 0.149 & 0.072 & 0.029 \\
\hline & 1.03 & 3.49 & 0.018 & -0.003 & 0.173 & 0.138 & 0.067 & 0.036 \\
\hline \multirow[t]{2}{*}{$97.5 \%$} & 1.06 & 4.06 & 0.010 & 0.010 & 0.268 & 0.238 & 0.145 & 0.085 \\
\hline & 1.18 & 4.05 & 0.047 & 0.027 & 0.258 & 0.190 & 0.116 & 0.078 \\
\hline
\end{tabular}

Table A1: Quantiles $(Q)$ of the summary statistics for the stock market scenario.

Note: For each quantile, the first row gives the results of the 5000 simulations runs of the model and the second row the results of the 5000 bootstrap samples of the S\&P 500 returns. $V$ stands for volatility, $H$ for the Hill estimator.

\begin{tabular}{|c|c|c|c|c|c|c|}
\hline \multirow[b]{2}{*}{$Q$} & \multirow[b]{2}{*}{$V$} & \multirow[b]{2}{*}{$H$} & \multicolumn{4}{|c|}{ Autocorrelations of $\left|r_{t}\right|$ at Lag } \\
\hline & & & 1 & 10 & 50 & 100 \\
\hline \multirow[t]{2}{*}{$2.5 \%$} & 0.64 & 3.73 & 0.087 & 0.063 & 0.011 & -0.005 \\
\hline & 0.62 & 3.62 & 0.033 & 0.070 & 0.010 & -0.013 \\
\hline \multirow[t]{2}{*}{$50.0 \%$} & 0.66 & 4.08 & 0.120 & 0.093 & 0.028 & 0.006 \\
\hline & 0.67 & 4.05 & 0.076 & 0.100 & 0.034 & 0.015 \\
\hline \multirow[t]{2}{*}{$97.5 \%$} & 0.68 & 4.43 & 0.160 & 0.131 & 0.055 & 0.024 \\
\hline & 0.72 & 4.62 & 0.117 & 0.130 & 0.060 & 0.044 \\
\hline
\end{tabular}

Table A2: Quantiles $(Q)$ of the summary statistics for the FX market scenario.

Note: The empirical returns are from the USD-DEM exchange rate. The quantiles of the autocorrelation coefficients for the raw returns are very similar to Table A1. 